\title{
New fractional integral inequalities via Caputo-Fabrizio operator and an open problem concerning an integral inequality
}

\author{
Gustavo Asumu MBoro Nchama \\ Universidad Nacional de Guinea Ecuatorial (UNGE), Malabo, Guinea Ecuatorial
}

Received: 11 November 2019, Accepted: 29 April 2020

Published online: 22 June 2020.

\begin{abstract}
In this paper, author introduces some new integral inequalities by using the Caputo-Fabrizio (CF) fractional integral and functions with the same sense of variation. Also an open problem concerning an integral inequality is discussed.
\end{abstract}

Keywords: Fractional integral inequalities, fractional integral operator, fractional Calculus, continuous function.

\section{Introduction}

A fractional derivative is just an operator which generalizes the ordinary derivative, such that if the fractional derivative is represented by the operator symbol $D^{\alpha}$ then, when $\alpha=n$, it coincides with the usual differential operator $D^{n}$ [16]. Its origin dates back to 1695 when L'Hopital raised by a letter to Leibniz the question of how the expression

$$
D^{n} u(t)=\frac{d^{n}}{d t^{n}} u(t)
$$

should be understood if $n$ was a real number [16]. Since then, the fractional derivative has become popular and useful due to its ability to describe some natural phenomena in numerous fields of engineering such as theory of viscoelasticity [3-5], study of the anomalous diffusion phenomenon [19-21], circuit theory [22-24], image processing [25, 26] and optimal control theory [12-15], among other applications. Various definition of fractional derivatives have been introduced [27-33]. In fact, the Grunwald-Letnikov fractional derivative, defined as a limit of a fractional order backward difference, is one of the first introduced fractional operators. Other definition which also plays a major role in Fractional Calculus is the Riemann-Liouville fractional derivative. The Caputo fractional derivative has also been defined via a modified Riemann-Liouville fractional derivative. This approach is useful for the formulation and solution of applied problems [29]. In 2015, Caputo and Fabrizio introduced a new fractional approach [31], which was born due to the necessity to describe a class of non-local systems which cannot be well described by classical local theories or by fractional models with singular kernel [31].

In recent years, many researchers have obtained integral inequalities using fractional integral operators [2, 5, 6, 10]. For example, in [5] appear fractional integral inequalities using Riemann-Liouville fractional integral:

\footnotetext{
*Corresponding author e-mail: becquerrr10@ hotmail.com
} 
Theorem 1. Let $f, g$ and $h$ be positive and continuous functions on $[0, \infty)$, such that

$$
(g(\tau)-g(\rho))\left(\frac{f(\rho)}{h(\rho)}-\frac{f(\tau)}{h(\tau)}\right) \geq 0 ; \quad \tau, \rho \in[0, t], \quad t>0
$$

then we have

$$
\frac{J^{\alpha}(f(t))}{J^{\alpha}(h(t))} \geq \frac{J^{\alpha}(g f(t))}{J^{\alpha}(g h(t))}
$$

for all $\alpha>0, t>0$.

Theorem 2. Let $f, g$ and $h$ be positive and continuous functions on $[0, \infty)$, such that

$$
(g(\tau)-g(\rho))\left(\frac{f(\rho)}{h(\rho)}-\frac{f(\tau)}{h(\tau)}\right) \geq 0 ; \quad \tau, \rho \in[0, t], \quad t>0
$$

then for all $\alpha>0, w, t>0$, we have

$$
\frac{J^{\alpha}(f(t)) \cdot J^{w}(g h(t))+J^{w}(f(t)) \cdot J^{\alpha}(g h(t))}{J^{\alpha}(h(t)) \cdot J^{w}(g f(t))+J^{w}(h(t)) \cdot J^{\alpha}(g f(t))} \geq 1 .
$$

Theorem 3. Let $f$ and $h$ be two positive continuous functions and $f \leq h$ on $[0, \infty)$. If $\frac{f}{h}$ is decreasing and $f$ is increasing on $[0, \infty)$, then for any $p \geq 1, \alpha>0, t>0$, the inequality

$$
\frac{J^{\alpha}(f(t))}{J^{\alpha}(h(t))} \geq \frac{J^{\alpha}\left(f^{p}(t)\right)}{J^{\alpha}\left(h^{p}(t)\right)},
$$

is valid.

Theorem 4. Let $f$ and $h$ be two positive continuous functions and $f \leq h$ on $[0, \infty)$. If $\frac{f}{h}$ is decreasing and $f$ is increasing on $[0, \infty)$, then for any $p \geq 1, \alpha>0, w>0, t>0$, we have

$$
\frac{J^{\alpha}(f(t)) \cdot J^{w}\left(h^{p}(t)\right)+J^{w}(f(t)) \cdot J^{\alpha}\left(h^{p}(t)\right)}{J^{\alpha}(h(t)) \cdot J^{w}\left(f^{p}(t)\right)+J^{w}(h(t)) \cdot J^{\alpha}\left(f^{p}(t)\right)} \geq 1 .
$$

In [7], it is established the following integral inequalities:

Theorem 5. Let $f(x) \geq 0$ be a continuous function on $[a, b]$ and satisfies $\left[(y-a)^{\alpha} \cdot f^{\alpha}(x)-(x-a)^{\alpha} \cdot f^{\alpha}(y)\right] \cdot\left[f^{\beta-\gamma}(x)-\right.$ $\left.f^{\beta-\gamma}(y)\right] \geq 0, \forall x, y \in[a, b]$ and $f^{\beta}(x) \leq f^{\gamma}(x), \forall x \in[a, b]$. Then for every positive real number $\alpha>0$ and $\beta \geq \gamma>0$ the inequality

$$
\frac{\int_{a}^{b} f^{\alpha+\beta}(x) d x}{\int_{a}^{b} f^{\alpha+\gamma}(x) d x} \geq \frac{\left(\int_{a}^{b}(x-a)^{\alpha} \cdot f^{\beta}(x) d x\right)^{\delta}}{\left(\int_{a}^{b}(x-a)^{\alpha} \cdot f^{\gamma}(x) d x\right)^{\lambda}},
$$

holds under each of the following conditions

(1) $\lambda=\delta=0$ and $\beta=\gamma, \forall x \in[a, b]$,

(2) $\lambda=\delta \in[1,+\infty), \forall x \in[a, b]$,

(3) If $\int_{x}^{b} f^{\beta}(t) d t \geq \frac{1}{(b-a)^{\alpha}}$ and $1 \leq \delta<1, \forall x \in[a, b]$,

(4) If $\int_{x}^{b} f^{\beta}(t) d t \leq \frac{1}{(b-a)^{\alpha}}$ and $1 \leq \lambda<\delta, \forall x \in[a, b]$.

Theorem 6. Let $f(x), g(x)>0$ continuous function on $[a, b]$ and satisfies

$$
\left[g^{\alpha}(y) f^{\alpha}(x)-g^{\alpha}(x) f^{\alpha}(y)\right] \cdot\left[f^{\beta-\gamma}(x)-f^{\beta-\gamma}(y)\right] \geq 0, \quad \forall x, y \in[a, b]
$$


and $f^{\beta}(x) \leq f^{\gamma}(x), \forall x \in[a, b]$. Then for every positive real number $\alpha>0$ and $\beta \geq \gamma>0$, the inequality

$$
\frac{\int_{a}^{b} f^{\alpha+\beta}(x) d x}{\int_{a}^{b} f^{\alpha+\gamma}(x) d x} \geq \frac{\left(\int_{a}^{b} g^{\alpha}(x) \cdot f^{\beta}(x) d x\right)^{\delta}}{\left(\int_{a}^{b} g^{\alpha}(x) \cdot f^{\gamma}(x) d x\right)^{\lambda}}
$$

holds under each of the following conditions

(1) $\lambda=\delta=0$ and $\beta=\gamma, \forall x \in[a, b]$,

(2) $\lambda=\delta \in[1,+\infty), \forall x \in[a, b]$,

(3) If $g^{\alpha}(a) \geq \frac{1}{(b-a) f^{\gamma}(a)}$ and $1 \leq \delta<\lambda$,

(4) If $g^{\alpha}(b) \leq \frac{1}{(b-a) f^{\beta}(b)}$ and $1 \leq \lambda<\delta$.

Also we can find some interesting integral inequalities in [11]:

Theorem 7. Let $\alpha>0, \mu>0, \delta>0, \beta>0, \gamma>0$ and let $f(t)$ be a positive and continuous function on $(0, \infty)$ such that

$$
\begin{aligned}
\left(\tau^{\mu}-\rho^{\mu}\right)\left(f^{\delta+\beta-\gamma}(\rho)-f^{\delta+\beta-\gamma}(\tau)\right) & \geq 0, \\
\left(\tau^{\mu}-\rho^{\mu}\right)\left(\frac{1}{f^{\delta}(\rho)}-\frac{1}{f^{\delta}(\tau)}\right) & \geq 0, \\
\left(f^{\beta-\gamma}(\tau)-f^{\beta-\gamma}(\rho)\right)\left(\frac{1}{f^{\delta}(\rho)}-\frac{1}{f^{\delta}(\tau)}\right) & \geq 0,
\end{aligned}
$$

for all $\tau, \rho \in(0, t]$. Then we have

$$
\frac{J^{\alpha}\left(f^{\delta+\beta}(t)\right)}{J^{\alpha}\left(f^{\delta+\gamma}(t)\right)} \geq \frac{J^{\alpha}\left(t^{\mu} f^{\beta}(t)\right)}{J^{\alpha}\left(t^{\mu} f^{\gamma}(t)\right)} .
$$

Theorem 8. Let $\alpha>0, \mu>0, \gamma>0, \delta>0, \beta>0, t>0$ and let $f(t)$ be a positive and continuous function on $(0, \infty)$ such that

$$
\left(f^{\beta-\gamma}(\tau)-f^{\beta-\gamma}(\rho)\right)\left(\frac{1}{f^{\delta}(\rho)}-\frac{1}{f^{\delta}(\tau)}\right) \geq 0
$$

for all $\tau, \rho \in(0, t]$. Then we have

$$
\frac{J^{\alpha}\left(t^{\mu} f^{\gamma}(t)\right)}{J^{\alpha}\left(t^{\mu} f^{\delta+\gamma}(t)\right)} \geq \frac{J^{\alpha}\left(t^{\mu} f^{\beta}(t)\right)}{J^{\alpha}\left(t^{\mu} f^{\delta+\beta}(t)\right)} .
$$

Theorem 9. Let $\alpha>0, \mu>0, \delta>0, \beta>0, \gamma>0$ and let $f(t)$ be a positive and continuous function on $(0, \infty)$ such that

$$
\begin{array}{r}
\left(\tau^{\mu}-\rho^{\mu}\right)\left(\frac{1}{f^{\delta}(\rho)}-\frac{1}{f^{\delta}(\tau)}\right) \geq 0, \\
\left(f^{\beta-\gamma}(\tau)-f^{\beta-\gamma}(\rho)\right)\left(\frac{1}{f^{\delta}(\rho)}-\frac{1}{f^{\delta}(\tau)}\right) \geq 0,
\end{array}
$$

for all $\tau, \rho \in(0, t]$. Then we have

$$
\frac{J^{\alpha}\left(f^{\gamma}(t)\right)}{J^{\alpha}\left(f^{\delta+\gamma}(t)\right)} \geq \frac{J^{\alpha}\left(t^{\mu} f^{\beta}(t)\right)}{J^{\alpha}\left(t^{\mu} f^{\delta+\beta}(t)\right)} .
$$

Theorem 10. Let $\alpha>0, \delta>0, \beta>0$ and $f(t)$ be a continuous function on $(0, \infty)$ such that $f(t) \geq t$ on $(0, \infty)$.

(a) If $0<\gamma<1$ and $J^{\alpha}\left(t^{\delta} f^{\beta}(t)\right) \geq 1$, then we have

$$
J^{\alpha}\left(f^{\delta+\beta}(t)\right) \geq\left(J^{\alpha}\left(t^{\delta} f^{\beta}(t)\right)\right)^{\gamma}
$$


(b) If $\gamma \geq 1$ and $0<J^{\alpha}\left(t^{\delta} f^{\beta}(t)\right)<1$, then we have

$$
J^{\alpha}\left(f^{\delta+\beta}(t)\right) \geq\left(J^{\alpha}\left(t^{\delta} f^{\beta}(t)\right)\right)^{\gamma}
$$

Theorem 11. Let $\alpha>0, \delta>0, \beta>0, \gamma>0,0<r<1, s \geq 1$ and let $f(t)$ be a positive and continuous function on (0, $\infty)$ such that

$$
\begin{aligned}
J^{\alpha}\left(t^{\delta} \cdot f^{\gamma}(t)\right) \geq 1, \quad J^{\alpha}\left(t^{\delta} \cdot f^{\beta}(t)\right) & \geq 1, \\
\left(\tau^{\mu}-\rho^{\mu}\right)\left(f^{\delta+\beta-\gamma}(\rho)-f^{\delta+\beta-\gamma}(\tau)\right) & \geq 0, \\
\left(\tau^{\mu}-\rho^{\mu}\right)\left(\frac{1}{f^{\delta}(\rho)}-\frac{1}{f^{\delta}(\tau)}\right) & \geq 0, \\
\left(f^{\beta-\gamma}(\tau)-f^{\beta-\gamma}(\rho)\right)\left(\frac{1}{f^{\delta}(\rho)}-\frac{1}{f^{\delta}(\tau)}\right) & \geq 0,
\end{aligned}
$$

for all $\tau, \rho \in(0, t]$. Then we have

$$
\frac{J^{\alpha}\left(f^{\delta+\beta}(t)\right)}{J^{\alpha}\left(f^{\delta+\gamma}(t)\right)} \geq \frac{\left(J^{\alpha}\left(t^{\delta} f^{\beta}(t)\right)\right)^{r}}{\left(J^{\alpha}\left(t^{\delta} f^{\gamma}(t)\right)\right)^{s}}
$$

And in [8], authors proved the following results:

Theorem 12. Let $f(x), g(x) \geq 0$ be continuous functions on $[a, b]$ and satisfy

$$
\left[g^{\alpha}(y) \cdot f^{\alpha}(x)-g^{\alpha}(x) \cdot f^{\alpha}(y)\right]\left[f^{\beta-\gamma}(x)-f^{\beta-\gamma}(y)\right] \geq 0, \forall x, y \in[a, b] .
$$

Then the inequality

$$
\frac{\int_{a}^{b} f^{\alpha+\beta}(x) d x}{\int_{a}^{b} f^{\alpha+\gamma}(x) d x} \geq \frac{\int_{a}^{b} g^{\alpha}(x) \cdot f^{\beta}(x) d x}{\int_{a}^{b} g^{\alpha}(x) f^{\gamma}(x) d x},
$$

holds for every positive real number $\alpha>0$ and $\beta \geq \gamma>0$.

Theorem 13. Let $f(x), h(x)>0$ be continuous functions on $[a, b]$ with $f(x) \leq h(x)$ for all $x$ and such that $\frac{f(x)}{h(x)}$ is decreasing and $f(x)$ increasing. Assume that $\varphi(x)$ is a convex function with $\varphi(0)=0$. Then the inequality

$$
\frac{\int_{a}^{b} f(x) d x}{\int_{a}^{b} h(x) d x} \geq \frac{\int_{a}^{b} \varphi(f(x)) d x}{\int_{a}^{b} \varphi(h(x)) d x}
$$

holds.

Theorem 14. Let $f(x), g(x), h(x)>0$ be continuous functions on $[a, b]$ with $f(x) \leq h(x)$ for all $x$ and such that $\frac{f(x)}{h(x)}$ is decreasing and $f(x), g(x)$ are increasing. Assume that $\varphi(x)$ is a convex function with $\varphi(0)=0$. Then the inequality

$$
\frac{\int_{a}^{b} f(x) d x}{\int_{a}^{b} h(x) d x} \geq \frac{\int_{a}^{b} \varphi(f(x)) g(x) d x}{\int_{a}^{b} \varphi(h(x)) g(x) d x},
$$

holds.

Next, they proposed the following open problems: 
Open problem 1. Under what conditions does the inequality

$$
\int_{a}^{b} f^{\alpha+\beta}(x) d x \geq\left(\int_{a}^{b}(x-a)^{\alpha} \cdot f^{\beta}(x) d x\right)^{\lambda},
$$

hold for $\alpha, \beta$ and $\lambda$ ?.

Open problem 2. Under what conditions does the inequality

$$
\frac{\int_{a}^{b} f^{\alpha+\beta}(x) d x}{\int_{a}^{b} f^{\alpha+\gamma}(x) d x} \geq \frac{\left(\int_{a}^{b}(x-a)^{\alpha} f^{\beta}(x) d x\right)^{\delta}}{\left(\int_{a}^{b}(x-a)^{\alpha} f^{\gamma}(x) d x\right)^{\lambda}},
$$

hold for $\alpha, \beta, \gamma, \delta$ and $\lambda$ ?

Open problem 3. Assume that $\phi(x)$ is a convex function with $\phi(0)=0$. Under what conditions does the inequality

$$
\frac{\int_{a}^{b} f(x) d x}{\int_{a}^{b} h(x) d x} \geq \frac{\left(\int_{a}^{b} \phi(f(x)) g(x) d x\right)^{\delta}}{\left(\int_{a}^{b} \phi(h(x)) g(x) d x\right)^{\lambda}}
$$

hold for $\delta$ and $\lambda$ ?

In literature few results have been obtained on properties of the Caputo-Fabrizio fractional integral $[10,18]$. Motivated from [1], the main purpose of this paper is to establish some new inequalities using Caputo-Fabrizio fractional integral. Also a solution to the open problem 1 is established. The paper has been organized as follows, in Section 2, we define basic concepts and definitions. In Section 3, we give the main results. The paper finalize with the conclusion in the section 4 .

\section{Basic Concepts and definitions}

Firstly, we give some necessary definitions and preliminaries of fractional calculus theory which are used further in this paper.

Definition 1. Let $\alpha>0$. The Riemann-Liouville fractional integral of order $\alpha$ of a function $f$ is defined by [29]

$$
J^{\alpha} f(t)=\frac{1}{\Gamma(\alpha)} \int_{0}^{t}(t-\tau)^{\alpha-1} f(\tau) d \tau .
$$

Definition 2. Let $0<\alpha<1$. The Caputo-Fabrizio fractional integral of order $\alpha$ of a function $f$ is defined by [9,31]

$$
I_{0 t}^{\alpha} f(t)=(1-\alpha) f(t)+\alpha \int_{0}^{t} f(\tau) d \tau
$$

Definition 3. Let $0<\alpha<1$ The Caputo-Fabrizio fractional derivative of order $\alpha$ of a function $f$ is defined by [9, 32]

$$
D_{a t}^{\alpha} f(t)=\frac{1}{1-\alpha} \int_{a}^{t} e^{-\frac{\alpha}{1-\alpha}(t-\tau)} f^{\prime}(\tau) d \tau .
$$

Definition 4. We say that two functions $f$ and $g$ have the same sense of variation on $[0, \infty)$ if

$$
(f(\tau)-f(\rho))(g(\tau)-g(\rho)) \geq 0, \quad \tau, \rho \in(0, t), t>0 .
$$


Note 1. Let $M>0, p \geq 1$ and $f, g$ be two positive functions on $[0, \infty)$. The inequality $\frac{f}{g} \geq M$ is equivalent to

$$
M^{p}(f+g)^{p} \leq(M+1)^{p} \cdot f^{p}
$$

because

$$
M^{p}(f+g)^{p} \geq(M+1)^{p} f^{p} \Leftrightarrow M(f+g) \geq(M+1) f \Leftrightarrow M g \geq f \Leftrightarrow M \geq \frac{f}{p} .
$$

Note 2. Let $m>0, p \geq 1$ and $f, g$ be two positive functions on $[0, \infty)$. The inequality $\frac{f}{g} \geq m$ is equivalent to

$$
(1 / m)^{p}(f+g)^{p} \geq(1 / m+1)^{p} \cdot g^{p}
$$

because

$$
\begin{aligned}
& (1 / m)^{p}(f+g)^{p} \geq(1 / m+1)^{p} g^{p} \Leftrightarrow(1 / m)(f+g) \geq(1 / m+1) g \Leftrightarrow \\
& \Leftrightarrow(1 / m) f \geq g \Leftrightarrow \frac{f}{p} \geq m .
\end{aligned}
$$

\section{Main Results}

In literature few results have been obtained on some fractional integral inequalities using Caputo-Fabrizio fractional integral [10]. The purpose of this section is to establish some new inequalities using the Caputo-Fabrizio fractional integral.

Theorem 15. Let $p \geq 1$ and let $f, g$ be two positive and continuous functions on $[0, \infty)$. If $0<m \leq \frac{f(\tau)}{g(\tau)} \leq M, \tau \in(0, t)$, then we have

$$
\left[I_{0 t}^{\alpha} f^{p}(t)\right]^{\frac{1}{p}}+\left[I_{0 t}^{\alpha} g^{p}(t)\right]^{\frac{1}{p}} \leq \frac{M(m+2)+1}{(M+1)(m+1)}\left[I_{0 t}^{\alpha}\left[(f+g)^{p}(t)\right]\right]^{\frac{1}{p}} .
$$

Proof. Using the condition $\frac{f(\tau)}{g(\tau)} \leq M, \tau \in(0, t), t>0$, we can write

$$
(M+1)^{p} \cdot f(\tau) \leq M^{p} \cdot(f+g)^{p}(\tau) .
$$

Multiplying both sides of (4) by $\alpha$, then integrating resulting identity with respect to $\tau$ from 0 to $t$, we get

$$
\begin{aligned}
& (M+1)^{p} \cdot\left[(1-\alpha) f^{p}(t)+\alpha \int_{0}^{t} f^{p}(s) d s-(1-\alpha) f^{p}(t)\right] \\
& \leq M^{p} \cdot\left[(1-\alpha)(f(t)+g(t))^{p}+\alpha \int_{0}^{t}(f(\tau)+g(\tau))^{p} d \tau-(1-\alpha)(f(t)+g(t))^{p}\right],
\end{aligned}
$$

which is equivalent to

$$
(M+1)^{p} I_{0 t}^{\alpha} f^{p}(t)+(1-\alpha) M^{p}[f+g]^{p} \leq M^{p} I_{0 t}^{\alpha}[f(t)+g(t)]^{p}+(M+1)^{p}(1-\alpha) f^{p}(t) .
$$

By using (1) in (5), follows

$$
(M+1)^{p} \cdot I_{0 t}^{\alpha} f^{p}(t) \leq M^{p} I_{0 t}^{\alpha}\left[(f+g)^{p}(t)\right]
$$

Hence, we can write

$$
\left[I_{0 t}^{\alpha} f^{p}(t)\right]^{\frac{1}{p}} \leq \frac{M}{M+1}\left[I_{0 t}^{\alpha}\left[(f+g)^{p}(t)\right]\right]^{\frac{1}{p}}
$$


On the other hand, from the condition $m \leq \frac{f(\tau)}{g(\tau)}$, we obtain

$$
\left(1+\frac{1}{m}\right) g(\tau) \leq \frac{1}{m}(f(\tau)+g(\tau)) .
$$

Therefore

$$
\left(1+\frac{1}{m}\right)^{p} g^{p}(\tau) \leq\left(\frac{1}{m}\right)^{p}(f(\tau)+g(\tau))^{p}
$$

Now, multiplying both sides of (7) by $\alpha$, then integrating resulting identity with respect to $\tau$ from 0 to $t$, we have the inequality

$$
\begin{aligned}
& \left(1+\frac{1}{m}\right)^{p}\left[(1-\alpha) g^{p}(t)+\alpha \int_{0}^{t} g^{p}(\tau) d \tau-(1-\alpha) g^{p}(t)\right] \leq \\
& \left(\frac{1}{m}\right)^{p}\left[(1-\alpha)(f(t)+g(t))^{p}+\alpha \int_{0}^{t}(f(\tau)+g(\tau))^{p} d \tau-(1-\alpha)(f(t)+g(t))^{p}\right],
\end{aligned}
$$

which implies

$$
\left(1+\frac{1}{m}\right)^{p} I_{0 t}^{\alpha} g^{p}+(1-\alpha)\left(\frac{1}{m}\right)^{p}(f+g)^{p} \leq\left(\frac{1}{m}\right)^{p} I_{0 t}^{\alpha}\left[(f+g)^{p}(t)\right]+(1-\alpha)\left(1+\frac{1}{m}\right)^{p} g^{p} .
$$

By using (2) into (8), yields

$$
\left(1+\frac{1}{m}\right)^{p} I_{0 t}^{\alpha} g^{p}(t) \leq\left(\frac{1}{m}\right)^{p} I_{0 t}^{\alpha}\left[(f+g)^{p}(t)\right]
$$

Hence, we can write

$$
\left[I_{0 t}^{\alpha} g^{p}(t)\right]^{\frac{1}{p}} \leq \frac{1}{m+1}\left[I_{0 t}^{\alpha}\left[(f+g)^{p}(t)\right]\right]^{\frac{1}{p}} .
$$

The inequality (3) follows on adding the inequalities (6) and (9).

Remark. Let $m>0, p>1, \frac{1}{p}+\frac{1}{q}=1$ and $f, g$ be two positive functions on $[0, \infty)$. The inequality $\frac{f}{g} \geq m$ is equivalent to

$$
m^{1 / p} g(t) \leq g^{1 / q} \cdot f^{1 / p}
$$

as

$$
\begin{aligned}
& \frac{f}{g} \geq m \Leftrightarrow m \leq \frac{f}{g} \Leftrightarrow m^{1 / p} \leq\left(\frac{f}{g}\right)^{1 / p} \Leftrightarrow m^{1 / p} \leq g^{-\frac{1}{p}} f^{\frac{1}{p}}, \\
& \Leftrightarrow m^{1 / p} \leq g^{\frac{1}{q}-1} f^{\frac{1}{p}} \Leftrightarrow m^{1 / p} g(t) \leq g^{1 / q} f^{1 / p} .
\end{aligned}
$$

Remark. In the same way, inequality $\frac{f}{g} \leq M$ is equivalent to

$$
M^{-1 / q} f(t) \leq[f(t)]^{1 / p}[g(t)]^{1 / q}
$$

Lemma 1. Let $0<\alpha<1, p>1, \frac{1}{p}+\frac{1}{q}=1$ and let $f$ and $g$ be two positive and continuous functions on $[0, \infty)$. If

$$
0<m \leq \frac{f(\tau)}{g(\tau)} \leq M<\infty, \quad \tau \in[0, t]
$$

then the inequality

$$
\left[I_{0 t}^{\alpha} f(t)\right]^{1 / p}\left[I_{0 t}^{\alpha} g(t)\right]^{1 / q} \leq\left(\frac{M}{m}\right)^{\frac{1}{p q}} I_{0 t}^{\alpha}\left[(f(t))^{\frac{1}{p}}(g(t))^{\frac{1}{q}}\right]
$$

holds. 
Proof. Since $\frac{f(\tau)}{g(\tau)} \leq M, \tau \in[0, t], t>0$, therefore

$$
[g(\tau)]^{1 / q} \geq M^{-1 / q}[f(\tau)]^{1 / q}
$$

and so

$$
[f(\tau)]^{1 / p}[g(\tau)]^{1 / q} \geq M^{-1 / q}[f(\tau)]^{1 / q}[f(\tau)]^{1 / p}=M^{-1 / q} f(\tau) .
$$

Integrating (13) with respect to $\tau$ from 0 to $t$, we have

$$
\begin{aligned}
& (1-\alpha)[f(t)]^{1 / p}[g(t)]^{1 / q}+\alpha \int_{0}^{t}[f(\tau)]^{1 / p}[g(\tau)]^{1 / q} d \tau-(1-\alpha)[f(\tau)]^{1 / p}[g(\tau)]^{1 / q} \\
& \geq M^{-1 / q}\left[(1-\alpha) f(t)+\alpha \int_{0}^{t} f(\tau) d \tau-(1-\alpha) f(t)\right] .
\end{aligned}
$$

That is

$$
\begin{aligned}
& I_{0 t}^{\alpha}\left[[f(t)]^{1 / p}[g(t)]^{1 / q}\right]+(1-\alpha) M^{-1 / q} f(t) \\
& \geq M^{-1 / q} I_{0 t}^{\alpha} f(t)+(1-\alpha)[f(t)]^{1 / p}[g(t)]^{1 / q} .
\end{aligned}
$$

By using (11) we obtain

$$
I_{0 t}^{\alpha}\left[[f(t)]^{1 / p}[g(t)]^{1 / q}\right] \geq M^{-1 / q} I_{0 t}^{\alpha} f(t)
$$

and consequently

$$
\left(I_{0 t}^{\alpha}\left[[f(t)]^{1 / p}[g(t)]^{1 / q}\right]\right)^{1 / p} \geq M^{-1 / p q}\left[I_{0 t}^{\alpha} f(t)\right]^{1 / p} .
$$

On the other hand, since $m g(\tau) \leq f(\tau), \tau \in[0, t], t>0$, then we have

$$
[f(\tau)]^{1 / p} \geq m^{1 / p}[g(\tau)]^{1 / p},
$$

and so

$$
[g(\tau)]^{1 / q}[f(\tau)]^{1 / p} \geq m^{1 / p}[g(\tau)]^{1 / p}[g(\tau)]^{1 / q}=m^{1 / p} g(\tau) .
$$

Now, multiplying both sides of (15) by $\alpha$, then integrating the resulting inequality with respect to $\tau$ over $(0, t)$, we obtain

$$
\begin{aligned}
& (1-\alpha)[g(t)]^{1 / q}[f(t)]^{1 / p}+\alpha \int_{0}^{t} g^{1 / q}(\tau) f^{1 / p}(\tau) d \tau-(1-\alpha)[g(t)]^{1 / q}[f(t)]^{1 / p} \\
& \geq m^{1 / p}\left[(1-\alpha) g(t)+\alpha \int_{0}^{t} g(\tau) d \tau-(1-\alpha) g(t)\right] .
\end{aligned}
$$

Therefore

$$
I_{0 t}^{\alpha}\left[[g(t)]^{1 / q}[f(t)]^{1 / p}\right]+(1-\alpha) m^{1 / p} g(t) \geq m^{1 / p} I_{0 t}^{\alpha} g(t)+(1-\alpha)[g(t)]^{1 / q}[f(t)]^{1 / p} .
$$

By using (10), we can write

$$
I_{0 t}^{\alpha}\left[[g(t)]^{1 / q}[f(t)]^{1 / p}\right] \geq m^{1 / p} I_{0 t}^{\alpha} g(t) .
$$

Hence, we obtain

$$
\left(I_{0 t}^{\alpha}\left[[g(t)]^{1 / q}[f(t)]^{1 / p}\right]\right)^{1 / q} \geq m^{1 / p q}\left(I_{0 t}^{\alpha} g(t)\right)^{1 / q} .
$$

Thanks to (14) and (16), we obtain (12). 
Lemma 2. Let $0<\alpha<1, p>1, \frac{1}{p}+\frac{1}{q}=1, f$ and $g$ be two positive and continuous functions on $[0, \infty)$. If

$$
0<m \leq \frac{(f(\tau))^{p}}{(g(\tau))^{q}} \leq M<\infty, \quad \tau \in[0, t],
$$

then we have

$$
\left[I_{0 t}^{\alpha} f^{p}(t)\right]^{1 / p}\left[I_{0 t}^{\alpha} g^{q}(t)\right]^{1 / q} \leq\left(\frac{M}{m}\right)^{\frac{1}{p q}} I_{0 t}^{\alpha}(f(t) g(t)) .
$$

Proof. Replacing $f(\tau)$ and $g(\tau)$ reswpectively by $(f(\tau))^{p}$ and $(g(\tau))^{q}, \tau \in[0, t], t>0$ in Lemma 1, we obtain (17).

Lemma 3. Let $0<\alpha<1, p>1, \frac{1}{p}+\frac{1}{q}=1$ and let fand $g$ be two positive and continuous functions on $[0, \infty)$. If

$$
0<m \leq \frac{f(\tau)}{g(\tau)} \leq M<\infty
$$

then

$$
I_{0 t}^{\alpha}\left[\frac{(f(t))^{p}}{(g(t))^{p / q}}\right] \leq\left(\frac{M}{m}\right)^{1 / q} \frac{\left(I_{0 t}^{\alpha} f(t)\right)^{p}}{\left(I_{0 t}^{\alpha} g(t)\right)^{p / q}}
$$

Proof. Using Lemma 1 we obtain

$$
\begin{aligned}
I_{0 t}^{\alpha}(f(t)) & =I_{0 t}^{\alpha}\left[\left(\frac{(f(t))^{p}}{(g(t))^{p / q}}\right)^{\frac{1}{p}}(g(t))^{\frac{1}{q}}\right] \\
& \geq\left(\frac{m}{M}\right)^{\frac{1}{p q}}\left[I_{0 t}^{\alpha} \frac{(f(t))^{p}}{(g(t))^{p / q}}\right]^{\frac{1}{p}}\left[I_{0 t}^{\alpha} g(t)\right]^{1 / q} .
\end{aligned}
$$

Hence, we can write

$$
\left[I_{0 t}^{\alpha}(f(t))\right]^{p} \geq\left(\frac{m}{M}\right)^{\frac{1}{q}}\left[I_{0 t}^{\alpha}\left[\frac{(f(t))^{p}}{(g(t))^{p / q}}\right]\right]\left[I_{0 t}^{\alpha} g(t)\right]^{p / q}
$$

Thanks to (19) we obtain (18).

Theorem 16. Let $0<\alpha<1, p>1, \frac{1}{p}+\frac{1}{q}=1$ and let $f$ be a positive and continuous function on $[0, \infty)$. If $0<m \leq f(\tau) \leq$ $M<\infty$ and

$$
I_{0 t}^{\alpha} f(t) \geq(1-\alpha+\alpha t)^{-\frac{p}{q}},
$$

then the inequality

$$
I_{0 t}^{\alpha}\left[(f(t))^{p}\right] \leq\left(\frac{M}{m}\right)^{1 / q}\left[I_{0 t}^{\alpha} f(t)\right]^{p+1},
$$

holds.

Proof. Using Lemma 3 and the condition (20), we obtain

$$
\begin{aligned}
I_{0 t}^{\alpha}\left[(f(t))^{p}\right] & =I_{0 t}^{\alpha}\left[\frac{(f(t))^{p}}{\left.(1)^{p / q}\right]}\right. \\
& \leq\left(\frac{M}{m}\right)^{1 / q} \cdot \frac{\left(I_{0 t}^{\alpha} f(t)\right)^{p}}{\left(I_{0 t}^{\alpha} 1\right)^{\frac{p}{q}}} \\
& \leq\left(\frac{M}{m}\right)^{1 / q}(1-\alpha+\alpha t)^{-\frac{p}{q}}\left(I_{0 t}^{\alpha} f(t)\right)^{p} \\
& \leq\left(\frac{M}{m}\right)^{1 / q}\left(I_{0 t}^{\alpha} f(t)\right)^{p+1},
\end{aligned}
$$

as required. 
The following theorem gives conditions under which the open problem 1 holds.

Theorem 17. Let $a, b \in \mathbb{R}(a<b)$ and $f(x)$ be a function such that $f(x) \geq x-a$ for all $x \in(a, b)$ and

$$
(1 /(\alpha+\beta+1)) \cdot(b-a)^{\alpha+\beta+1}>1,
$$

for $\alpha, \beta$ and $\gamma$ positive real numbers with $0<\gamma<1$. Then

$$
\int_{a}^{b} f^{\alpha+\beta}(x) d x \geq\left(\int_{a}^{b}(x-a)^{\alpha} \cdot f^{\beta}(x) d x\right)^{\gamma}
$$

Proof. From $f(x) \geq x-a>0$, it is easy to see that

$$
\begin{gathered}
(f(x))^{\alpha+\beta} \geq(x-a)^{\alpha+\beta}>0 \\
(f(x))^{\beta} \geq(x-a)^{\beta}>0 \\
(f(x))^{\alpha} \geq(x-a)^{\alpha}>0
\end{gathered}
$$

On the one hand, by using inequalities (21) and (23), we obtain

$$
\int_{a}^{b}(f(x))^{\alpha+\beta} d x \geq \int_{a}^{b}(x-a)^{\alpha+\beta} d x=(1 /(\alpha+\beta+1)) \cdot(b-a)^{\alpha+\beta+1}>1 .
$$

On the other hand, from (24) and (21), we obtain

$$
\begin{aligned}
\int_{a}^{b}(x-a)^{\alpha} \cdot f^{\beta}(x) d x & \geq \int_{a}^{b}(x-a)^{\alpha} \cdot(x-a)^{\beta} d x=\int_{a}^{b}(x-a)^{\alpha+\beta} d x \\
& =(1 /(\alpha+\beta+1)) \cdot(b-a)^{\alpha+\beta+1}>1 .
\end{aligned}
$$

Moreover, from (25) and (27), yields

$$
\int_{a}^{b} f^{\alpha+\beta}(x) d x=\int_{a}^{b} f^{\alpha}(x) \cdot f^{\beta}(x) d x \geq \int_{a}^{b}(x-a)^{\alpha} \cdot f^{\beta}(x) d x>1 .
$$

Combining (28) with the fact that $0<\gamma<1$, we obtain

$$
\begin{aligned}
\int_{a}^{b} f^{\alpha+\beta}(x) d x & =\int_{a}^{b} f^{\alpha}(x) \cdot f^{\beta}(x) d x \\
& \geq \int_{a}^{b}(x-a)^{\alpha} \cdot f^{\beta}(x) d x \geq\left(\int_{a}^{b}(x-a)^{\alpha} \cdot f^{\beta}(x) d x\right)^{\gamma}
\end{aligned}
$$

From (29), we obtain (22).

\section{Conclusion}

In this paper, we have used the Caputo-Fabrizio fractional integral to develop some interesting fractional inequalities. These results have been obtained with the help of functions with the same sense of variation. Also an open problem concerning an integral inequality has been discussed. As a future work, author is planning to use these inequalities to prove the existence and uniqueness of some ordinary differential equations containing the Caputo-Fabrizio operator. 


\section{Acknowledgements}

This work is supported by Universidad Nacional de Guinea Ecuatorial (UNGE) and Universidad de La Habana (UH). The author would like to thank the anonymous reviewers for their valuable suggestions that helped improve the presentation of this paper.

\section{Competing interests}

The authors declare that they have no competing interests.

\section{Authors' contributions}

All authors have contributed to all parts of the article. All authors read and approved the final manuscript.

\section{References}

[1] A. Anber, Z. Dahmani and B. Bendoukha, New Integral Inequalities of Feng Qi Type via Riemann-Liouville Fractional Integration, Ser. Math. Inform (2012), vol. 27, pp. 159-165.

[2] V. L. Chinchane and D. B. Pachpatte, New Fractional Inequalities Involving Saigo Fractional Integral Operator, Mathematical Sciences Letters (2014), vol. 3, pp. 133-135.

[3] M. Houas and M. Bezziou, On Some Fractional Integral Inequalities Involving Generalized Riemann-Liouville Fractional Integral Operator, Med. J. Model. Simul. (2016), pp. 59-65.

[4] M. Aldhaifallah, M. Tomar, K. S. Nisar and S. D. Purohit, Some new inequalities for $(k, s)$-fractional integrals, J. Nonlinear Sci. Appl. (2016), pp. 5376-5379.

[5] Z. Dahmani and H. Metakkel El Ard, Generalizations of Some Integral Inequalities Using Riemann-Liouville Operator. Int. J. Open Problems Compt. Math. (2011), vol. 4, pp. 42-44.

[6] Z. Dahmani, The Riemann-Liouville Operator to Generate Some New Inequalities, International Journal of Nonlinear Science (2011), vol. 12, pp. 453-455.

[7] A. Kashuri, R. Liko, A. Fundo and M. Ramosaco, Open Problem Related to integral inequalities, Journal of Inequalities and Special Functions (2016), vol. 7, pp. 115-118.

[8] W. Liu, Q. A. Ngo and V. N. Huy, Several Interesting integral Inequalities, Journal of Mathematical Inequalities (2009), vol. 3, pp. 203-211.

[9] N. Al-Salti, E. Karimov and S. Kerbal, Boundary-value problems for fractional heat equation involving Caputo-Fabrizio derivative, New Trends in Mathematical Sciences (2016), vol. 4, pp. 79-80.

[10] G. A. M. Nchama, A. L. Mecías and M. R. Ricard, The Caputo-Fabrizio fractional integral to generate some new inequalities, Information Sciences Letters (2019), vol. 8, pp. 73-80. 
[11] G. A. M. Nchama, On open problems concerning Riemann-Liouville fractional integral inequality, Med. J. Model. Simul. (2019), vol. 11, pp. 001-008.

[12] D. Baleanu, A. Jajarmi, S. S. Sajjadi and D. Mozyrska, A new fractional model and optimal control of a tumor-immune surveillance with non-singular derivative operator, Chaos (2019), vol. 29, 083127.

[13] A. Jajarmi, S. Arshad and D. Baleanu, A new fractional modelling and control strategy for the outbreak of dengue fever, Physica A (2019) vol. 535, 122524.

[14] A. Jajarmi, D. Baleanu, S. S. Sajjadi and J. H. Asad, A new feature of the fractional Euler-Lagrange equations for a coupled oscillator using a nonsingular operator approach, Frontiers in Physics (2019), vol. 7, 00196.

[15] A. Jajarmi, B. Ghanbari and Dumitru Baleanu, A new and efficient numerical method for the fractional modelling and optimal control of diabetes and tuberculosis co-existence, Chaos (2019), vol. 29, 093111.

[16] D. Baleanu, K. Diethelm, E. Escalas and J. J. Trujillo, Fractional Calculus: Models and numerical methods, Series on Complexity, Nonlinearity and Chaos (2012), vol. 3.

[17] B. M. Vinagre and C. A. Monje, Introducción al Control Fraccionario, Revista Iberoamericana de Automática e Informática Industrial (2006), vol. 3, pp. 5-23.

[18] G. A. M. Nchama, Properties of Caputo-Fabrizio fractional operators, New Trends in Mathematical Sciences (2020), vol. 8, pp. $1-25$.

[19] C. Ramírez, V. Astorga, H. Nuñez, A. Jaques and R. Simpson, Anomalous diffusion based on fractional calculus approach applied to drying analysis of apple slices: The effects of relative humidity and temperature, Food Process Engineering (2017), Article DOI: 10.1111/jfpe.12549.

[20] M. Concezzi and R. Spigler, Identifying the Fractional Orders in Anomalous Diffusion Models from Real Data, Fractal Fract. (2018), vol. 2, doi:10.3390/fractalfract2010014.

[21] X. Liang, F. Gao, C. B. Zhou, Z. Wang and X. J. Yang, An anomalous diffusion model based on a new general fractional operator with the Mittag-Leffler function of Wiman type, Advances in Difference Equations (2018), https://doi.org/10.1186/s13662-018$1478-1$.

[22] A. M. F. Andrade, E.G. Lima and C.A. Dartora, An introduction to fractional calculus and its Applications in Electric Circuits, Revista Brasileira de Ensino de Física (2018), vol. 40, e3314.

[23] V. F. M. Delgado, J. F. G. Aguilar and M. A. T. Hernandez, Analytical solutions of electrical circuits described by fractional conformable derivatives in Liouville-Caputo sense, International Journal of Electronics and Communications (2017), https://doi.org/10.1016/j.aeue.2017.12.031.

[24] R. Sikora, Fractional derivatives in electrical circuit theory critical remarks, Archives of Electrical Engineering (2017), vol. 66, pp. $155-163$.

[25] J. Yu, R. Zhai, S. Zhou and L. Tan, Image Denoising Based on Adaptive Fractional Order with Improved PM Model, Mathematical Problems in Engineering (2018), Article ID 9620754. 
[26] A. Nandal, H. G. Rosales, A. Dhaka, J. M. C. Padilla, J.I.G. Tejada, C.E.G. Tejada, F.J.M. Ruiz and C.G. Valdivia, Image Edge Detection Using Fractional Calculus with Feature and Contrast Enhancement, Circuits System Signal Process (2018), https://doi.org/10.1007/s00034-018-0751-6.

[27] J. Sabatier, O. P. Agrawal and J. A. Tenreiro Machado, Advanced in Fractional Calculus: Theoretical Developments and Applications in Physics and Engineering, Springer (2007).

[28] A. A. Kilbas, H. M. Srivastava and J. J. Trujillo, Theory and Applications of Fractional Differential Equations, North Holland Mathematics Studies (2006), vol. 204.

[29] I. Polubny, Fractional Differential Equations, Academic Press (1999), New York.

[30] K. B. Odham and J. Spanier, The Fractional Calculus, Academic Press (1984), New York.

[31] M. Caputo and M. Fabrizio, A new Definition of Fractional Derivative without Singular Kernel, Progr. Fract. Differ. Appl. (2015), vol. 1, pp. 73-85.

[32] J. Losada and J. J. Nieto, Properties of a New Fractional Derivative without Singular Kernel, Progr. Fract. Differ. Appl. (2015), vol. 1, pp. 87-92.

[33] A. Atangana and D. Baleanu, New fractional derivatives with nonlocal and non-singular kernel: Theory and application to heat transfer model, Thermal Science (2016), doi:10.2298/TSCI160111018A. 